\title{
Origami Bird Simulator: A Teaching Resource Linking Natural Selection and Speciation
}

\author{
Takahiro Yamanoi ${ }^{1 *}$ and Watal M Iwasaki
}

\begin{abstract}
The origami bird is a resource for teaching natural selection. A previous study incorporated the Gamete Mutation Box into the original protocol to assist in understanding the link between DNA mutation and natural selection. Having revised the protocol, we developed computer simulation software, called the Origami Bird Simulator (OBS), for teaching the connection between these two processes and speciation. In this paper, we investigate the effectiveness of the protocol modification and integrating OBS into the protocol of previous study. OBS enables students to witness the speciation process in allopatric origami bird populations through geographic isolation and following reproductive isolation. Results of tests before and after the students' experiment suggest that this new protocol enhances their understanding of the linkages between mutation, natural selection, and speciation.
\end{abstract}

Keywords: Evolution education, Japan, Computer simulation, Reproductive isolation, Genetic drift

\section{Background}

Evolution is one of the core concepts in understanding modern biology. Several researchers, however, have reported that understanding evolutionary mechanisms is difficult for secondary school and university students, for whom many misconceptions exist (Gregory 2009; Yamanoi et al. 2011). One of the main issues is that evolution is a complex subject that requires the ability to integrate knowledge of basic concepts such as DNA, natural selection, and speciation, across multiple scales of time, space, and biological organization (Speth et al. 2009). Although research studies on evolution education have attempted to mitigate these obstacles, most have focused only on natural selection (Catley 2006). Thus, techniques to assist understanding the connection between mutation and speciation are still lacking. Furthermore, the most recent version of the "course of study" for high school biology in Japan (Ministry of Education, Science, Sports and

\footnotetext{
*Correspondence: yamanoit@fc.hakuoh.ac.jp

1 Laboratory of Science Education, Faculty of Education, Hakuoh

University, 1117 Daigyoji, Oyama, Tochigi 323-8585, Japan

Full list of author information is available at the end of the article
}

Culture 2009) requires that the process by which mutations and natural selection lead to speciation is taught.

Various teaching resources for secondary school students aimed at evolutionary mechanisms have been developed in several countries (Alters and Alters 2001; Yamanoi et al. 2012a, b). These resources include physical simulations (e.g., Welch 1993; Christensen-Dalsgaard and Kanneworff 2008; Burton and Dobson 2009) and computer simulations (e.g., Speth et al. 2009; Jones and Laughlin 2010; Royer and Schultheis 2014) that are useful in students' investigative activities. Although computer programs can simulate the speciation process in a short time, most existing material has not taken advantage of this and has instead focused solely on microevolutionary processes. Development of teaching resources that incorporate both micro- and macroevolutionary processes is highly desirable.

Origami bird is a teaching resource for natural selection with physical simulation developed by Westerling (1992). Although students are also expected to learn about the process that produces intraspecies variations by mutation through the experiment, they may not understand mutation from a molecular basis because the precise DNA alteration process is not included in the protocol. 
Yamanoi et al. (2012a) tried to incorporate the DNA mutation process into the original protocol and reported that the enhanced origami bird protocol does promote an understanding of the link between DNA mutation and natural selection amongst Japanese high school students.

In this study, we first modified the enhanced protocol to connect the DNA mutation and natural selection processes smoothly. Then, we developed the computer simulation software, "Origami Bird Simulator" (OBS), to incorporate the speciation process. We expect that this modification and addition should improve students' comprehension of the entire process involving these evolutionary mechanisms by presenting the link between DNA mutation and speciation. The effectiveness of this protocol was confirmed by comparing the responses of Japanese high school students on questionnaires completed before and after participating in the experiment.

\section{Methods}

\section{Introduction of "Origami Bird"}

The origami bird as a teaching resource for natural selection was developed by Westerling (1992). Students learn about natural selection by making origami birds (from paper) and throwing them by hand, as one would paper airplanes. As this fictitious bird supposedly lives in arid regions of North Africa, only those birds that can fly the long distances between oases live long enough to breed successfully. The effects of mutations on their phenotypes are determined by a coin flip and a dice throw; the coin flip determines which part of the bird's wings is affected by the mutation, while the dice throw determines how the mutation affects the wing. Morphological changes often affect the bird's ability to fly long distances; only the individual with the longest flying distance in a particular generation survives and breeds.

Yamanoi et al. (2012a) modified this protocol to suit the learning of Japanese high school students. In this protocol, DNA alteration that occurs in a bird's offspring is determined by rotating the roulette wheels of the Gamete Mutation Box (GMB), which consists of a short DNA sequence and two roulette wheels. The DNA includes five genes (despite each gene having three bases, the roulette wheels change only one of the three bases) that determine a bird's phenotype (the number of clips, wing color, distances between the forewing and hindwing, and wing size). One of the roulette wheels of the GMB determines the gene that mutates, while the other one determines the base after substitution. After DNA alteration, the student can determine the type of phenotype change caused by the mutation using a "mutation table". For example, if the GMB roulette wheels indicate a mutation of the fourth gene (affecting the size of the wings) from $\mathrm{G}$ to $\mathrm{A}$, the mutation table dictates a $1 \mathrm{~cm}$ enlargement of both wings. Students create three birds per generation by rotating the GMB's roulette wheels three times per generation to create three offspring. After folding the three birds, they throw them in the corridor adjacent to the classroom to investigate how they "fly". Only the most adaptive bird achieving the longest flight distance of the three survives and reproduces in that generation.

Yamanoi et al. (2012a) evaluated the educational effect of their protocol using pre- and post-tests (27 questions on the definition of evolution, natural selection, and mutation) and free comment after the student experiment. They concluded that the students considered mutation correctly as random DNA alteration, which enabled them to understand evolution by linking changes at the DNA level to those at the phenotype level, and rejected teleological thoughts, which are a widespread misconception that Japanese high school students retain even after they have finished high school (Yamanoi et al. 2011). This improved protocol is valuable and useful for evolution education because it enhances students' understanding of the link between altering the DNA and natural selection. However, their protocol did not include any mention of speciation, thereby denying the students the opportunity of learning the role of mutation in speciation through the experiment.

\section{Modifications to the Protocol of Yamanoi et al. (2012a)}

To improve understanding of speciation through geographic isolation and microevolution, we revised the protocol to include two environments with different directional selection. In the first environment with a small number of oases, natural selection favors the individual with the longest flight distance, i.e., one that can reach a scarce oasis. Only this direction of selection was considered in the previous protocol. In the other environment, rich in oases, the individual with the shortest flight distance is selected because it can reach an oasis with the lowest cost. These alternative selections make a difference in the average flight distance of groups after four generations as expected by Yamanoi et al. (2012a).

The old protocol has a further drawback in that phenotypes do not have a one-to-one correspondence with genotypes. In other words, one genotype can have different phenotypic outcomes depending on the history of mutations. For example, an A-to-G mutation on the locus of the number of clips causes a one clip increase, whereas mutating from $A$ to $G$ through $C$ causes an increase of two clips. To address this problem, we created a "genotype-phenotype mapping table" to replace the "mutation table" in the old protocol (Fig. 1). Phenotypes, therefore, depend only on the current states of genotypes.

Although it is known that many mutations are neutral, or almost neutral, with a very small effect on fitness 


\begin{tabular}{|c|c|c|c|c|c|c|c|c|c|c|c|}
\hline \multicolumn{12}{|c|}{ Genotype-Phenotype Mapping Table (effects of mutation on phenotype) } \\
\hline \multirow{2}{*}{$\begin{array}{c}\text { Gene } 1 \\
\text { DNA }\end{array}$} & \multicolumn{2}{|c|}{ Number of clips } & \multirow[b]{2}{*}{ phenotype } & \multirow{2}{*}{$\begin{array}{c}\underline{\text { Gene } 2} \\
\text { DNA }\end{array}$} & \multicolumn{2}{|c|}{ Color of the wings } & \multirow[b]{2}{*}{ phenotype } & \multirow{2}{*}{$\begin{array}{r}\text { Gene } 3 \\
\text { DNA }\end{array}$} & \multicolumn{2}{|c|}{ Position of the wings } & \multirow[b]{2}{*}{ phenotype } \\
\hline & mRNA & amino acid & & & mRNA & amino acid & & & mRNA & amino acid & \\
\hline AGA & UCU & serine & 1 & TTA & AAU & asparagine & blue & GAA & CUU & leucine & $4 \mathrm{~cm}$ from each edge \\
\hline TGA & ACU & threonine & 1 & TTT & AAA & lysine & red & GTA & CAU & histidine & $2 \mathrm{~cm}$ from each edge \\
\hline GGA & CCU & proline & 2 & TTG & $A A C$ & asparagine & blue & GGA & $\mathrm{CCU}$ & proline & $4 \mathrm{~cm}$ from each edge \\
\hline CGA & GCU & alanine & 0 & TTC & AAG & lysine & red & GCA & CGU & arginine & $3 \mathrm{~cm}$ from each edge \\
\hline \multicolumn{12}{|c|}{ Gene 4 Size of forewing } \\
\hline DNA & mRNA & amino acid & \multicolumn{3}{|c|}{ phenotype } & & & & & & \\
\hline CAA & GUU & valine & \multicolumn{3}{|c|}{ width $4 \mathrm{~cm}$ length $30 \mathrm{~cm}$} & & & & & & \\
\hline CTA & GAU & asparagine acid & \multicolumn{3}{|c|}{ width $2 \mathrm{~cm}$ length $20 \mathrm{~cm}$} & & & & & & \\
\hline CGA & GCU & alanine & \multicolumn{3}{|c|}{ width $3 \mathrm{~cm}$ length $26 \mathrm{~cm}$} & & & & & & \\
\hline CCA & GGU & glycine & \multicolumn{3}{|c|}{ width $1 \mathrm{~cm}$ length $18 \mathrm{~cm}$} & & & & & & \\
\hline Gene 5 & Size of hin & dwing & & & & & & & & & \\
\hline DNA & mRNA & amino acid & \multicolumn{3}{|c|}{ phenotype } & & & & & & \\
\hline AGC & UCG & serine & \multicolumn{3}{|c|}{ width $2 \mathrm{~cm}$ length $20 \mathrm{~cm}$} & & & & & & \\
\hline TGC & ACG & threonine & \multicolumn{3}{|c|}{ width $1 \mathrm{~cm}$ length $18 \mathrm{~cm}$} & & & & & & \\
\hline$G G C$ & CCG & proline & \multicolumn{3}{|c|}{ width $4 \mathrm{~cm}$ length $30 \mathrm{~cm}$} & & & & & & \\
\hline CGC & GCG & alanine & \multicolumn{3}{|c|}{ width $3 \mathrm{~cm}$ length $26 \mathrm{~cm}$} & & & & & & \\
\hline
\end{tabular}

Fig. 1 Genotype-phenotype mapping table. A base depicted in red denotes the changeable base in each gene through GMB roulettes. A sequence shaded gray denotes the sequence of the P-th generation in each gene.

(Futuyma 2009), in this teaching resource, we only considered influential genes (e.g., the HOX gene) affecting body plans of the embryo. Therefore, even a single base substitution could cause extensive changes in a bird's morphology in this system. The teacher discussed this with the students during the experiment.

At the start of the experiment, the teacher explained to the students that a population of origami birds had split into two separate populations owing to geographic isolation, and different phenotypes were favored in the two environments with different numbers of oases, as explained above. The numbers of groups in each environment were adjusted to be almost equal through communication with other groups. After the fourth iteration of the hand-throwing experiment, students wrote their flight distance history on the blackboard in front of the class.

The worksheet for filling in the results was updated to incorporate the genotype-phenotype map and evolutionary trajectories in the two different environments (Fig. 2). The main differences between the protocol of Yamanoi et al. and our revised protocol are illustrated in Fig. 3.

\section{Development of Origami Bird Simulator}

Although it was expected that students would learn how directional selection shapes the evolutionary trajectory of phenotypes through the hand-throwing experiment, it was difficult to incorporate the concept of speciation into the protocol. Another problem arose in that each step of the experiment took such a long time that only a few generations could be observed in a class. To solve these problems, we developed a computer program that simulates and visualizes the evolution and speciation of origami birds. The software was designed to be used after the hand-throwing experiment, to help students visualize what could be observed if many iterations of the experiment covering many generations were conducted.

The OBS starts at the time an origami bird population splits into two separate populations. The application window has three tabs. In the first two tabs, the user observes the evolution of the two respective populations. The user can alter the parameters (environment, mutation rate, population size, and observation period) for each tab. The environment denotes the abundance of oases and can be chosen from three options: rich, medium, and 
a Front side of worksheet (redacted version)

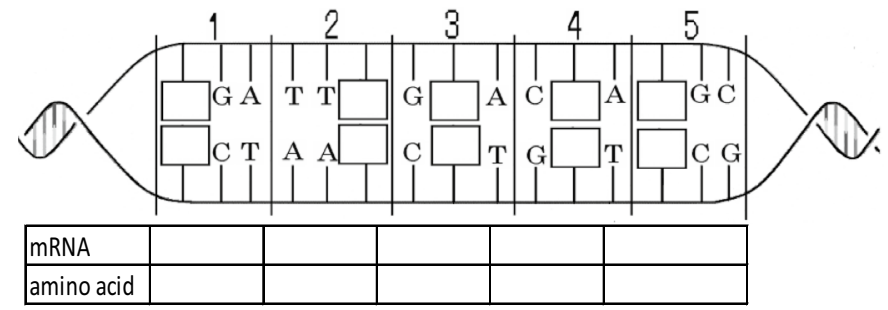

\begin{tabular}{|c|c|c|c|c|}
\hline forewing & width & $\mathrm{cm}$ & length & $\mathrm{cm}$ \\
\hline hindwing & width & $\mathrm{cm}$ & length & $\mathrm{cm}$ \\
\hline wing color & & & $\begin{array}{l}\text { number of } \\
\text { clips }\end{array}$ & \\
\hline \multicolumn{2}{|c|}{$\begin{array}{c}\text { wing position: distance } \\
\text { from each edge }\end{array}$} & $\mathrm{cm}$ & & \\
\hline $\begin{array}{c}\text { maximum } \\
\text { flight } \\
\text { distance }\end{array}$ & & $\mathrm{cm}$ & & \\
\hline
\end{tabular}

\section{b Rear side of worksheet (redacted version)}

\begin{tabular}{|c|c|c|c|c|c|c|c|}
\hline \multicolumn{7}{|c|}{ The environment with rich oasis } \\
\hline \multicolumn{7}{|c|}{ (Only the individual with the longest flight distance will survive and reproduce) } \\
\hline & group 1 & group 2 & group 3 & group 4 & group 5 & average \\
\hline$P$ & & & & & & $\mathrm{~cm}$ \\
\hline$F_{1}$ & & & & & & $\mathrm{~cm}$ \\
\hline$F_{2}$ & & & & & & $\mathrm{~cm}$ \\
\hline$F_{3}$ & & & & & & $\mathrm{~cm}$ \\
\hline$F_{4}$ & & & & & & $\mathrm{~cm}$ \\
\hline \multicolumn{7}{|c|}{ The environment with poor oasis } & \\
\hline (Only the individual with the shortest flight distance will survive and reproduce) \\
\hline$P$ & group 1 & group 2 & group 3 & group 4 & group 5 & average \\
\hline$P$ & & & & & & $\mathrm{~cm}$ \\
\hline$F_{1}$ & & & & & & $\mathrm{~cm}$ \\
\hline$F_{2}$ & & & & & & $\mathrm{~cm}$ \\
\hline$F_{3}$ & & & & & & $\mathrm{~cm}$ \\
\hline$F_{4}$ & & & & & & $\mathrm{~cm}$ \\
\hline
\end{tabular}

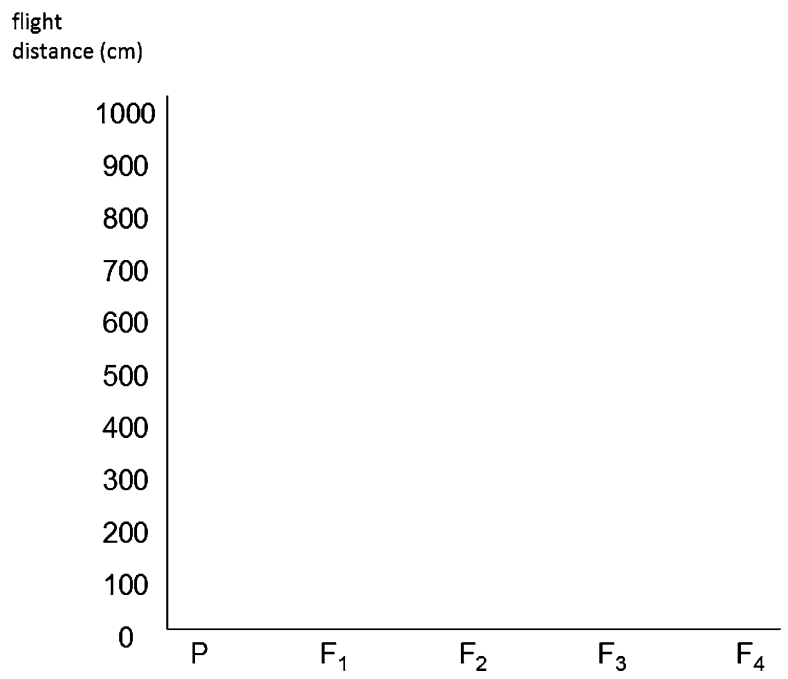

Fig. 2 Result handout. a Students considered the table (Fig. 1), then filled in changes in mRNA and amino acid other than changes in the DNA base and phenotype. b After the hand throwing experiment, they filled in the flight distances of each generation per group, and then plotted a graph based on the average flight distance in each environment per generation calculated from all groups'scores.

poor. Figure 4a illustrates an example where the oasispoor environment is chosen. When the "Lock" button is pressed, the parameters are locked and the simulation gets ready to start. Ten individuals, randomly chosen from the population, are displayed on the right-hand side. After the evolutionary simulation, a test subject for the crossing experiment is selected by clicking on the individual in this panel. The user repeats these steps in the second tab (Fig. 4b shows an example where the user chose the oasis-rich environment). In the "Crossing Experiment" tab, the selected individuals from the two populations are displayed. The pair attempts to mate when the "Cross" button is pressed. Children are born if they succeed (Fig. 4c); otherwise, the animation for copulation failure is displayed (Fig. 4d). Copulation success depends on the phenotypic differences between mating 


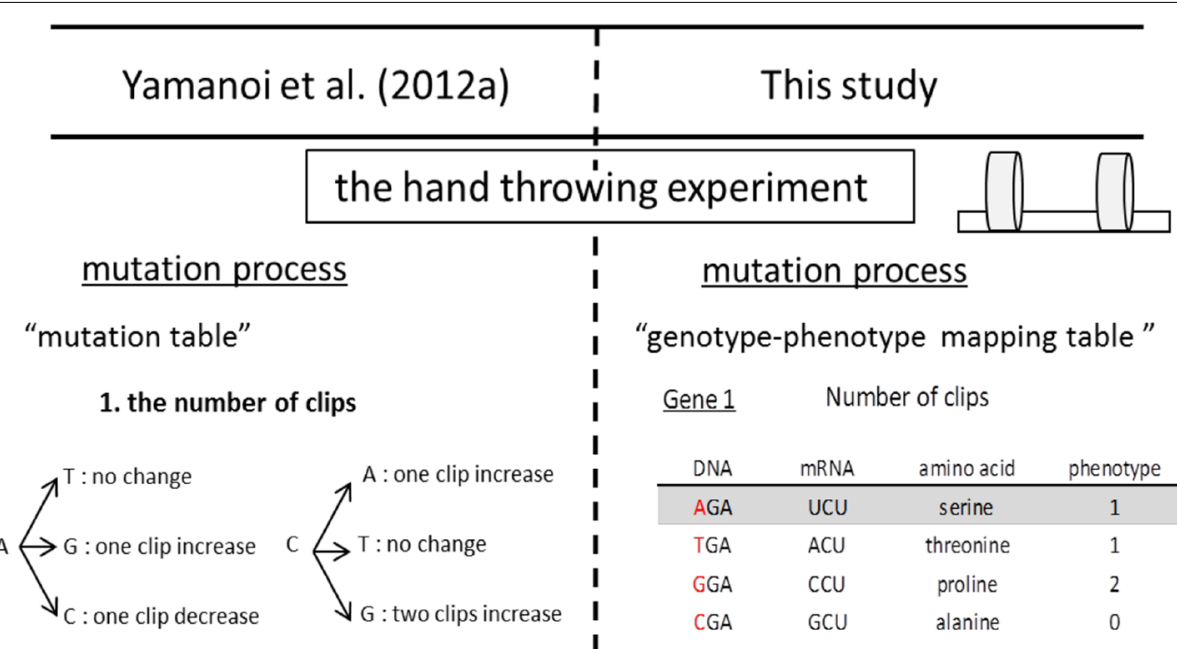

Mutation to a specific base can have different phenotypic effects depending on the original base that was mutated.

selection process

oasis: poor

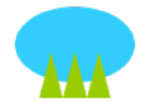

We prepared one environment where only the bird achieving the longest flight distance survives and reproduces in that generation.

Only the sequence of the gene determines the phenotype.

\section{selection process}

oasis: poor
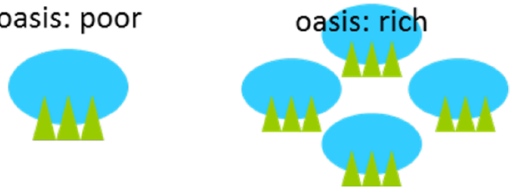

We prepared two environments where selection pressure was different.

\section{the computer simulation} experiment with OBS

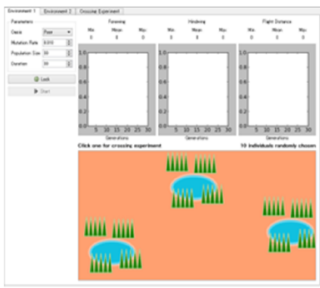

Fig. 3 Differences between the protocol of Yamanoi et al. and our revised protocol.

partners, the criteria of which are described later. Two individuals from different environments are expected to have such dissimilar phenotypes that prezygotic isolation occurs.

\section{Definitions of Genotype and Phenotype}

An individual has a forewing and a hindwing, each of which has two loci with four alleles (nucleotides). The wing sizes are calculated by scaling them to integer numbers (e.g. AA: 0, AT: 1, TA:4, CG: 14) and by averaging the values of two chromosomes. For example, an individual with genotype [AATT, GGCC] develops a forewing from heterozygotic AA:GG with size $(0+10) / 2=5$. When this individual mates and reproduces, possible zygotes are not only [AATT] and [GGCC], but also [AATC] and [GATT] because of recombination. Other traits such as the number of clips and wing color have not yet been implemented. 


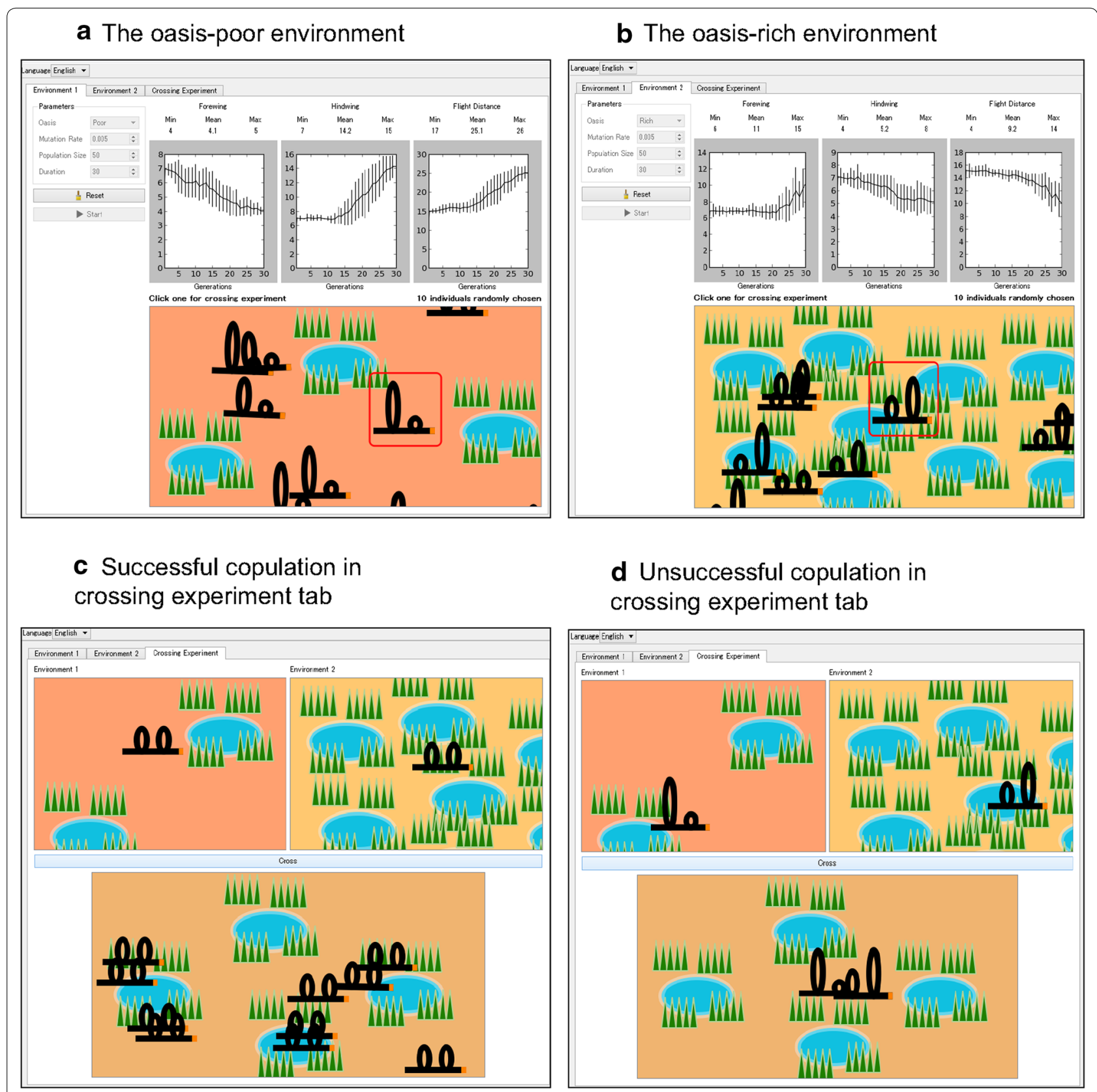

Fig. 4 How to use OBS. In "Environment 1" (a) and "Environment 2" (b), users select the number of oases (rich or poor), enter values for the three parameters (population size, generation time, mutation rate) and then click "start" to begin the simulation. In this figure, a represents an oasis-poor environment, and $\mathbf{b}$ an oasis-rich environment, with the graph illustrating how the sizes of the forewing and hindwing and the flight distance change through 30 generations. Users can click on an individual to select it for copulation in its environment, and the individual will be surrounded by a square $(\mathbf{a}, \mathbf{b})$; then the selected individuals are displayed on the crossing experiment tab $(\mathbf{c}, \mathbf{d})$. In this tab, after clicking the "cross" button, users can observe whether the two individuals can copulate. If they can, an animation indicating successful copulation and their offspring is displayed (c). If they cannot copulate, the animation shows unsuccessful copulation in that they approach each other and try to copulate, but separate immediately (d). Although we used the Japanese version of OBS for the student experiment, this figure depicts the English version. Users can easily select their language (English or Japanese) on the "language tab" at the top of the OBS window.

\section{Flight Distance}

We assume that the flight distance is determined only by the size of the wings, calculated as (hindwing - forewing) +15 . In other words, the flight distance increases with increasing hindwing size or decreasing forewing size. This equation is based on the 
observation in the experiments by Yamanoi (2010), where the students were encouraged to throw an origami bird with constant power to minimize the effect of the throwing motion.

\section{Fitness Function}

We assume that the optimal flight distance increases with fewer oases in the environment. The Gaussian function is adopted as a fitness function and normalized so that an individual with the optimal flight distance obtains the maximum fitness 1.0 (Lande 1975; Wagner 1989; Johnson and Barton 2005). The fitness of individual $i$ is defined as

$$
W_{i}=\exp \left(-\frac{\left(x_{i}-x_{o}\right)^{2}}{2 \sigma^{2}}\right)
$$

where $x_{i}$ is the flight distance divided by the maximum distance $30, x_{o}$ is the optimal value in each environment (0.03 for oasis-rich, 0.5 for intermediate, and 0.97 for oasis-poor), and $\sigma$ is fixed at 0.5 (Fig. 5).

\section{Mutation Rate}

The default mutation rate is $10^{-2}$ per locus per generation. Although this is much higher than the estimated values in real organisms, it is necessary to shorten the waiting time to get an evolutionary outcome. Besides, it makes no qualitative difference to our purpose. The same value is assigned to the recombination rate. The user can alter the value between $10^{-3}$ and $10^{-1}$.

\section{Mating and Reproduction}

A population with $N$ individuals means $N / 2$ parent pairs. Each pair lays 20 eggs if they can copulate; otherwise,

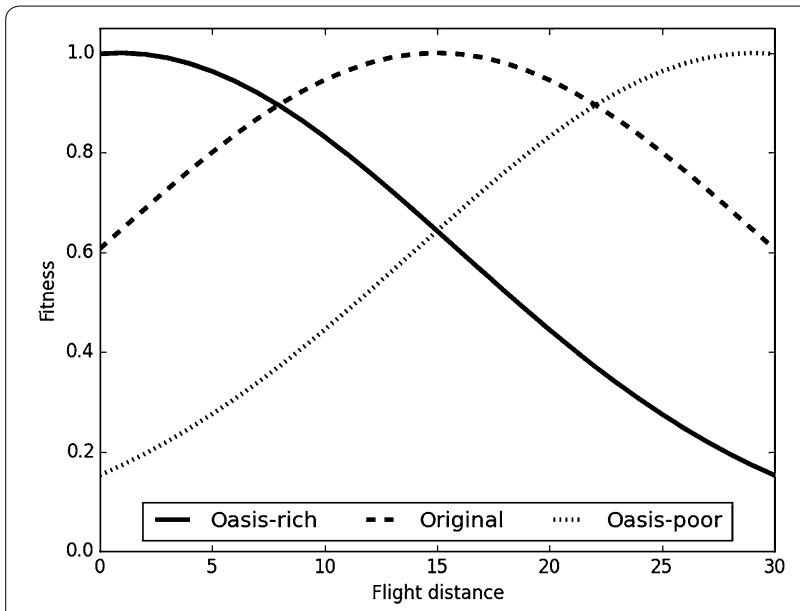

Fig. 5 Relationship between fitness and flight distance in OBS. The optimal flight distance is determined by the abundance of oases in an environment. Solid, dashed, and dotted lines denote oasis-rich, original (intermediate), and oasis-poor environments, respectively. there are no eggs. Populations are isolated from each other and have no gene flows. Population size is constant and $N$ children survive to the next reproduction. Roulette selection is performed based on the relative fitness of each individual. The survival probability of individual $i$ is expressed as

$$
P_{i}=\frac{W_{i}}{\sum_{j} W_{j}} .
$$

\section{Reproductive Isolation}

We assume post-mating, prezygotic barriers. Copulation success in each generation and crossing experiment depends on the differences in wing sizes of the mating partners. First, the differences in forewing and hindwing sizes both need to be smaller than five. A male attempts to put his head through the partner's hindwing from behind if her hindwing is larger than her forewing (Fig. 6a). In this case, his forewing (Fig. 4c left) needs to be smaller than his partner's hindwing (Fig. 4c right). On the contrary, a male attempts to put the partner's tail through his forewing from behind if her hindwing is smaller than her forewing (Fig. 6b). Here, the male's forewing needs to be larger than his partner's hindwing.

\section{Student Experiment and Analysis of its Educational Effect}

We conducted the student experiment in three Japanese high schools in 2011 (A high school in Tokyo, $N=11$, male:female ratio $=6: 5$; $\mathrm{B}$ high school in Tochigi, $N=18$, male:female $=10: 8 ; C$ high school in Kyoto, $N=45$, male:female $=15: 30$; total number of students $=74$ ). Student experiments at high schools A and B were overseen by an author, whereas that at high school $\mathrm{C}$ was administered by the biology teacher at the school. (We explained the teaching method using our new protocol to him before the experiment.) The students were biology majors and had completed a course in evolutionary concepts (e.g., natural selection, mutation, microevolution, macroevolution, and biological species). It took two class sessions $(45 \mathrm{~min} \times 2)$ to complete the process.

In the first session (about $70 \mathrm{~min}$ ), we performed the origami bird experiment (by hand throwing) using the new protocol. In the closing stage of this session, the teacher emphasized that evolutionary trajectories varied depending on the environment by showing the aggregated results for all the groups; see Figure 10 in Yamanoi et al. 2012a.

In the next session (20 min), the teacher performed the computer simulation experiment using OBS, projecting the simulation process on the screen in front of the class. Before the simulation, the teacher asked the students "What will happen to populations of origami birds in each environment after 30 generations? Will speciation occur?" Then, the teacher explained that OBS was built 

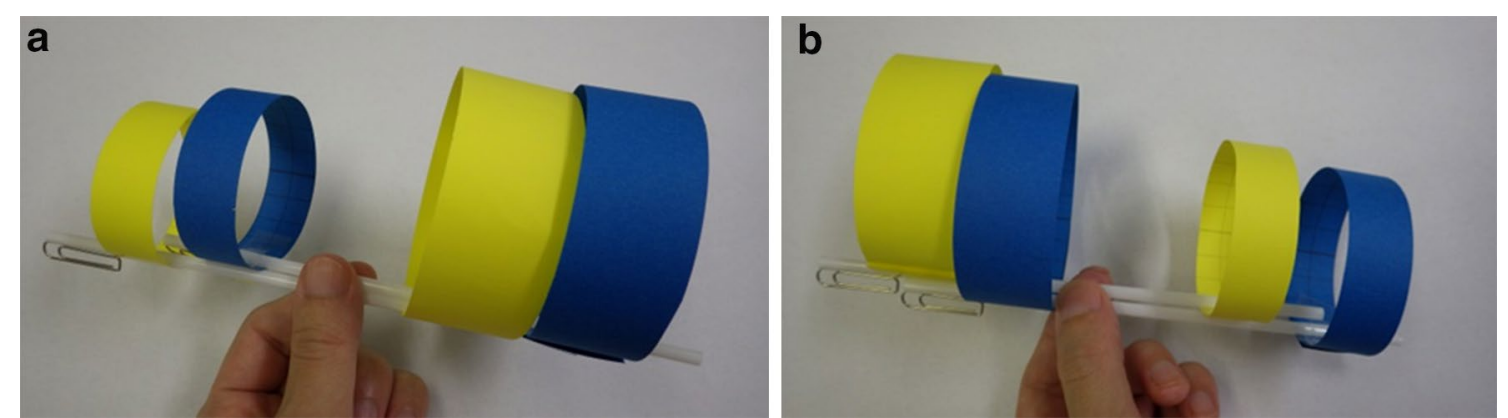

Fig. 6 Copulation methods in origami birds. Yellow individuals are female, blue ones are male. If the differences in the forewing and hindwing sizes of the two partners are small, copulation will occur in OBS. The copulation method (a or b) depends on the shapes of the partners' wings. We assume that if the female's forewing is smaller than her hindwing, the male attempts to put his head through his partner's hindwing from behind (a). Alternatively, the male attempts to put the partner's tail through his forewing from behind if the female's forewing is larger than her hindwing (b)

based on similar settings (e.g., adaptive wing shape in each environment) to those in the hand throwing origami bird experiment and that pairs could copulate if their wing sizes were similar. The teacher performed simulations under two conditions that differed only with respect to population size: large (population size 50, mutation rate 0.005 , observation period 30 ), and small (population size 10 , mutation rate 0.005 , observation period 30). The simulation was repeated two or three times for each condition. Teachers selected two or three pairs and crossed these birds in the 30th generation separately for each condition. In the first case, the teacher showed the simulation result where a bird from an oasis-rich environment and one from an oasis-poor environment could not copulate after 30 generations and explained that these populations had become different species based on the biological species concept. In the second case, the teacher showed that sometimes two populations did not reach speciation and explained that the intensity of directional natural selection was weak and the effect of genetic drift became perceptible with a small population size.

We used the questionnaire method to compare the students' understanding before and after the experiment. Our learning objective was that students should be able to understand evolutionary mechanisms by presenting the link between DNA mutation and speciation. We evaluated their understanding thereof by asking to what extent DNA mutation affects speciation (Table 1). Each question is related to either the link between DNA mutation and genetic diversity (Q.4), phenotype change (Q.3, 5), natural selection (Q.6), speciation (Q.7), or species diversity (Q.8). Furthermore, as the premise of these questions, we also asked for a definition of mutation $(\mathrm{Q} .1,2)$. The validity of our questionnaire was confirmed by a preliminary survey of graduate students and post-doctoral fellows studying evolution in a Japanese
Table 1 Questions included in the pre- and post-experiment questionnaire

\author{
1. Mutation means the changes in DNA sequences of individuals \\ 2. Mutation means speciation \\ 3. There are DNA changes that do not affect the phenotype \\ 4. DNA alterations are needed to cause genetic diversity \\ 5. DNA alterations can cause phenotypic variations \\ 6. DNA alterations are necessary for the evolution by natural selection \\ 7. DNA alterations are necessary for speciation \\ 8. DNA alterations are needed to cause species diversity
}

national graduate school $(\mathrm{n}=16$, average years studying evolution: 5.6 years). Subjects were given five choices for each question (strongly agree, agree, neutral, disagree, and completely disagree). We adopted eight questions that received consistent responses; two questions about the definition of evolution and the role of genetic drift in the process of speciation were excluded based on the results of our preliminary survey. Over $80 \%$ of the subjects answered "strongly agree" or "agree" for the eight questions, except for question 2, which was the only question requiring reversed responses. The numbers of "strongly agree" and "agree" responses (\%) for each question were: Q.1 62.5, 25; Q.3 87.5, 12.5; Q.4 81.3, 12.5; Q.5 87.5, 6.3; Q.6 68.8, 12.5; Q.7 75.0, 18.8; and Q.8 75.0, 18.8 . For question 2 , over $80 \%$ of the subjects answered "disagree" (12.5\%) or "completely disagree" (87.5\%). The reliability of our questionnaire was confirmed by re-testing the same subjects. Approximately $80 \%$ of the responses were identical to those in the first test. The pre-experiment questionnaire was completed before the handthrowing experiment, while the post-experiment one was administered after the computer simulation experiment. In this way, we investigated the combined educational 
effects of the hand-throwing experiment with the new protocol and the computer simulation experiment using OBS. The pre- and post-experiment questionnaires were almost identical, except that two questions were added to the post-experiment questionnaire. One was "Did your understanding of DNA, evolution and biodiversity change after the experiment?" Responses could be selected from five options (changed significantly, changed slightly, unaware of any change, changed minimally, did not change). If a student's response was "changed significantly" or "changed slightly," we also asked him/her to elaborate on the reasons. The other question was "Please write down your impressions about the experiment."

\section{Results}

An increase in "strongly agree" responses in several questions was noted after the experiment (Table 2); the number of "strongly agree" responses to question 7 , in particular, showed a $30 \%$ increase in the post-test. To confirm whether each student response changed to a more positive one after the experiment, we assumed each nominal scale to be an equal interval and scored each response as follows: strongly agree $=$ five points, agree $=$ four points, neutral $=$ three points, disagree $=$ two points, and completely disagree $=$ one point. Note that this scoring was reversed for question 2. The scores increased significantly after the experiment for seven out of the ten questions $(\mathrm{p}<0.05$, paired $\mathrm{t}$ test, questions 1, 3, 6-8; Fig. 7). No questions achieved a lower score for the post-test.

Most students (71 \%) responded that their understanding of DNA, evolution, and biodiversity changed after the experiment (Fig. 8). The free description of the improvement included comments on the effect of population size and time on the speciation process $(n=6)$, genetic drift $(n=5)$, evolutionary mechanisms $(n=4)$, the link between the mutation and speciation processes $(n=3)$, stochasticity of the simulation $(n=3)$, and noting that DNA alterations do not always cause a change in phenotype $(n=2)$, mutation is not synonymous with speciation $(n=2)$, and speciation can occur more easily than the student thought $(n=2)$. Other minor comments are listed below: "Before this experiment, I had associated mutations with white snakes and limbless animals, not with speciation. Now I realize that mutations will lead to speciation after tens of thousands of years" (High school A, female); "I understood that genetic drift has stronger effects on evolution than natural selection does" (High school C, male).

Student impressions $(n>1)$ given as free comments included the following: interesting $(n=20)$, promotes understanding about evolutionary mechanisms $(n=12)$, surprised that random DNA alteration causes adaptation $(n=3)$, want to use OBS by myself and run simulations under many other conditions $(n=3)$, surprised that bird morphology can affect its flight distance $(n=2)$, easy to understand $(\mathrm{n}=2)$, and began to feel that evolution is understandable $(n=2)$.

\section{Discussion}

We determined the combined educational effects of the hand-throwing experiment with the new protocol and the computer simulation experiment using OBS by comparing the students' responses before and after the experiment.

Table 2 Student responses before and after the experiment

\begin{tabular}{|c|c|c|c|c|c|c|c|c|}
\hline & \multicolumn{2}{|l|}{ Q.1 } & \multicolumn{2}{|l|}{ Q.2 } & \multicolumn{2}{|l|}{ Q.3 } & \multicolumn{2}{|l|}{ Q.4 } \\
\hline & Pre & Post & Pre & Post & Pre & Post & Pre & Post \\
\hline Strongly agree & 53.5 & 77.5 & 22.9 & 29.6 & 62.9 & 81.4 & 71.8 & 69.0 \\
\hline Agree & 29.6 & 15.5 & 14.3 & 15.5 & 14.3 & 8.6 & 16.9 & 25.4 \\
\hline Neutral & 11.3 & 2.8 & 25.7 & 22.5 & 11.4 & 5.7 & 11.3 & 4.2 \\
\hline Disagree & 2.8 & 2.8 & 20.0 & 7.0 & 2.9 & 1.4 & 0.0 & 1.4 \\
\hline \multirow[t]{3}{*}{ Completely disagree } & 2.8 & 2.8 & 17.1 & 25.4 & 8.6 & 2.9 & 0.0 & 0.0 \\
\hline & \multicolumn{2}{|l|}{ Q.5 } & \multicolumn{2}{|l|}{ Q.6 } & \multicolumn{2}{|l|}{ Q.7 } & \multicolumn{2}{|l|}{ Q.8 } \\
\hline & Pre & Post & Pre & Post & Pre & Post & Pre & Post \\
\hline Strongly agree & 70.8 & 80.6 & 52.8 & 66.7 & 40.0 & 70.0 & 64.8 & 77.8 \\
\hline Agree & 20.8 & 15.3 & 25.0 & 22.2 & 31.4 & 20.0 & 18.3 & 18.1 \\
\hline Neutral & 6.9 & 2.8 & 11.1 & 8.3 & 17.1 & 8.6 & 9.9 & 4.2 \\
\hline Disagree & 0.0 & 0.0 & 5.6 & 0.0 & 7.1 & 1.4 & 5.6 & 0.0 \\
\hline Completely disagree & 1.4 & 1.4 & 5.6 & 2.8 & 4.3 & 0.0 & 1.4 & 0.0 \\
\hline
\end{tabular}

Numbers 1-8 denote the question numbers and decimal numbers in the table indicate the number of responses (\%). The correct response for each question is italicized. 


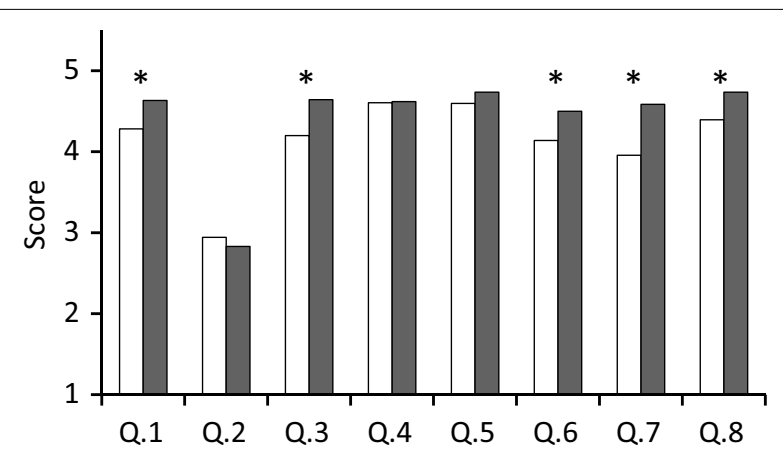

Fig. 7 Changes in the average scores for individual questions before and after the experiment. Asterisks indicate a significant difference at the 0.05 level.

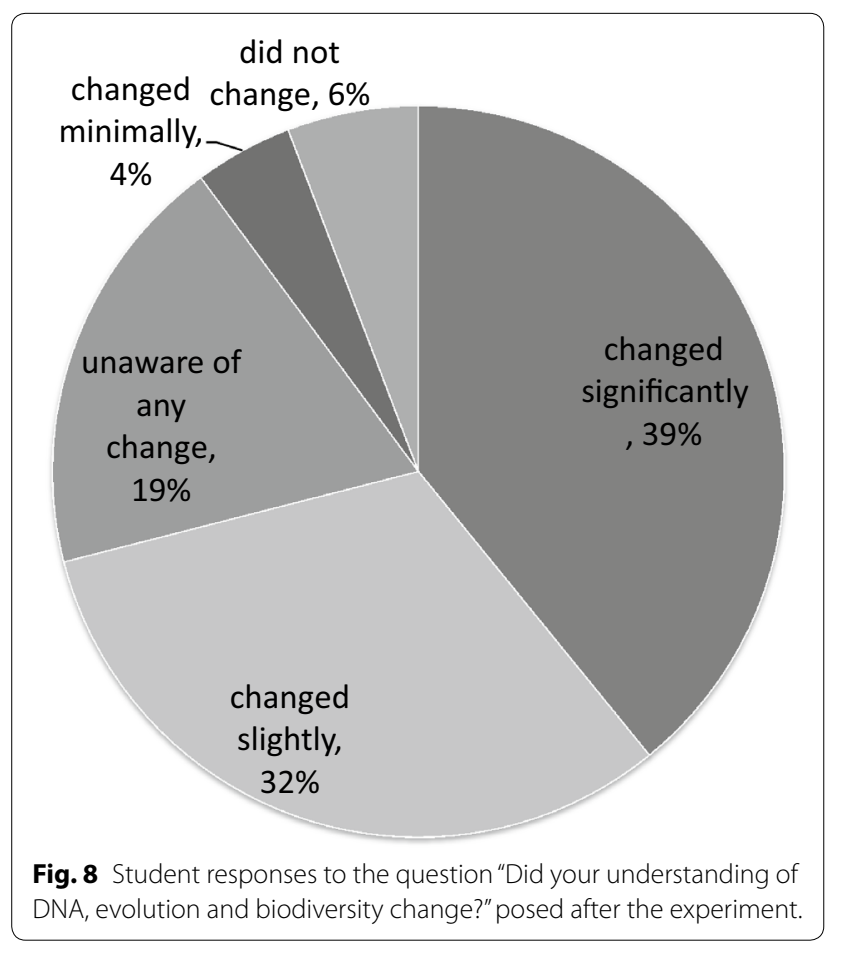

The improved scores for questions $6-8$ and the fact that most students $(71 \%)$ responded that their understanding of DNA, evolution, and biodiversity improved after the experiment (Figs. 7, 8) indicate that the experiment enhanced students' understanding of the link between altering the DNA, natural selection, speciation, and species diversity. The scores for question 7, in particular, increased significantly after the experiment. Since content regarding the link between altering the DNA and speciation was introduced into the old protocol by this research, this significant increase is considered to be derived from the introduction of the revised protocol of the hand-throwing experiment and the computer simulation experiment using OBS.
Free format comments on the improved understanding included references to the effect of population size and time on the speciation process, genetic drift, and the stochasticity of the simulation. Since the content related to these comments was included in the computer simulation experiment with OBS, these improvements are considered to have occurred as a result of the simulation software. However, to reveal the precise educational effects of the experiment using OBS, a questionnaire survey needs to be administered between the hand-throwing and computer simulation experiments and the responses compared.

From the scores for question 2, many students seemed to confuse mutation with speciation as reported in previous studies on the origami bird (Yamanoi 2008, 2010). Some students seemed to improve their understanding through the experiment as the proportion of "completely disagree" responses increased from 17.1 to $25.4 \%$. However, teachers should be careful to explain the meanings of each term before they perform the experiments using our protocol. The scores for questions 4 and 5 did not increase, presumably because the score before the experiment was already high (4.61 and 4.60, respectively).

Most of these results support the hypothesis that the students' understanding improved as a result of the experiment. We expect that if students could operate the OBS themselves, they would understand the evolutionary process even more because of curiosity. However, the origami bird experiment using the OBS simulation could result in some misunderstandings: some students erroneously thought that speciation occurs in a short time, and they overestimated the power of genetic drift on the evolutionary mechanism. Teachers should be aware that the speciation process that occurs over hundreds of thousands of years is taught as an event that lasts only $90 \mathrm{~min}$ for descriptive purposes. Combined usage of other teaching resources focusing on the timescale of speciation (e.g., Metzger 2011) could be effective. In a future experiment, students will run the simulation with three population sizes (large: $n=100$, medium: $\mathrm{n}=30$, small: $n=10$ ). This should enable them to understand that the effect of natural selection due to genetic drift is noticeable if the population size is large, whereas it is not perceptible when the population size is small. We preliminarily performed this experiment for seven groups under each condition. The average ratio of successive mating was $26 \%$ (large), $37 \%$ (medium), $33 \%$ (small), which supported our prediction. Although the average ratio of successive mating with a small population (33\%) was lower than that with a medium population size (37 \%), the ratios with a small population fluctuated more, indicating that genetic drift masks the effects of natural selection. As there are only a few teaching resources for understanding the balance of 
natural selection and genetic drift (e.g., Russo and Voloch 2012), the OBS will be a useful addition.

\section{Conclusion}

We modified the origami bird protocol with hand throwing (Westerling 1992; Yamanoi et al. 2012a, b) and developed the OBS computer simulation software to demonstrate evolutionary mechanisms from DNA alteration to speciation. Modification of the enhanced protocol and introduction of the OBS simulations improved the students' understanding of these evolutionary processes. We intend to alter the experiment to include student execution of the OBS. If students were able to operate the simulation directly, they would gain a more intuitive understanding of evolutionary mechanisms by freely changing the parameters of the OBS out of curiosity. Our assessment method was not adequate for measuring the diversity and frequency of the key concepts included in the students' answers about evolution (Nehm and Schonfeld 2008; Speth et al. 2009). In future experiments, we intend confirming whether students apply the principles of mutation, natural selection, and speciation to explain how real organisms' populations change over time using a variety of assessment methods such as open-response instruments. The source code and executable program of OBS are available at https://github.com/heavywatal/ori$\mathrm{bir} /$. We expect that the OBS will be used for evolution education in secondary schools and universities in many countries around the globe.

\section{Authors' Contributions}

TY conceived the study, performed the analyses, interpreted the results, and drafted the manuscript. WMI constructed the computer simulations, interpreted the results, and helped draft the manuscript. Both authors read and approved the final manuscript.

\section{Author details}

${ }^{1}$ Laboratory of Science Education, Faculty of Education, Hakuoh University, 1117 Daigyoji, Oyama, Tochigi 323-8585, Japan. ${ }^{2}$ Department of Evolutionary Studies of Biosystems, SOKENDAI (Graduate University for Advanced Studies), Hayama, Kanagawa 240-0193, Japan.

\begin{abstract}
Acknowledgements
We thank the teachers and students for their participation in our experiment, and the graduate school students and post-doctoral fellows of SOKENDAI (Graduate University for Advanced Studies) for their cooperation in the preliminary questionnaire survey. Thanks are also due to $\mathrm{H}$. Tanaka, O. Sakura, N. Mizushima, and M. Takemura for their insightful comments. This study was partially supported by a Grant-in-Aid for Scientific Research (B) \#22330253 (MT) and a Grant-in-Aid for Young Scientists (B) \# 25870669 (TY) from the Ministry of Education, Culture, Sports, Science and Technology of Japan.
\end{abstract}

\section{Compliance with Ethical Guidelines}

\section{Competing Interests}

The authors declare that they have no competing interests

\section{References}

Alters, B. J., \& Alters, S. M. (2001). Defending evolution: a guide to the creation/ evolution controversy. Sudbury: Jones and Bartlett Publishers.

Burton, S. R., \& Dobson, C. (2009). Spork \& beans: addressing evolutionary misconceptions. The American Biology Teacher, 71(2), 89-91.

Catley, K. M. (2006). Darwin's missing link: a new paradigm for evolution education. Science Education, 90(5), 767-783.

Christensen-Dalsgaard, J., \& Kanneworff, M. (2008). Evolution in Lego ${ }^{\circledR}$ : a physical simulation of adaptation by natural selection. Evolution: Education and Outreach, 2, 518-526.

Futuyma, D. J. (2009). Evolution (2nd ed.). Massachusetts: Sinauer Associates inc.

Gregory, T. R. (2009). Understanding natural selection: essential concepts and common misconceptions. Evolution: Education \& Outreach, 2, 156-175.

Johnson, T., \& Barton, N. (2005). Theoretical models of selection and mutation on quantitative traits. Philosophical Transactions of the Royal Society B-Biological Sciences, 360(1459), 1411-1425

Jones, C. T., \& Laughlin, T. F. (2010). PopGen fishbowl: a free online simulation model of microevolutionary processes. The American Biology Teacher, 72(2), 100-103.

Lande, R. (1975). The maintenance of genetic variability by mutation in a polygenic character with linked loci. Genetical Research, 26(3), 221-235.

Metzger, K. J. (2011). Helping students conceptualize species divergence events using the online tool "TimeTree: the timescale of life". The American Biology Teacher, 73(2), 106-108.

Ministry of Education, Science, Sports and Culture. (2009). Course of study for secondary schools in Japan. Tokyo: Printing bureau, Ministry of Finance. (in Japanese)

Nehm, R. H., \& Schonfeld, I. S. (2008). Measuring knowledge of natural selection: a comparison of the CINS, an open-response instrument, and an oral interview. Journal of Research in Science Teaching, 45, 1131-1160.

Royer, A. M., \& Schultheis, E. H. (2014). Evolving better cars: teaching evolution by natural selection with a digital inquiry activity. The American Biology Teacher, 76(4), 259-264.

Russo, C. A. M., \& Voloch, C. M. (2012). Beads and dice in a genetic drift exercise. Evolution: Education \& Outreach, 5, 494-500.

Speth, E. B., Long, T. M., Pennock, R. T., \& Ebert-May, D. (2009). Using Avida-ED for teaching and learning about evolution in undergraduate introductory biology courses. Evolution: Education \& Outreach, 2, 415-428.

Wagner, G. P. (1989). Multivariate mutation-selection balance with constrained pleiotropic effects. Genetics, 122(1), 223-234.

Welch, L. A. (1993). A model of microevolution in action. The American Biology Teacher, 55(6), 362-365.

Westerling, KE (1992). http://www.indiana.edu/ ensiweb/lessons/origami. html. Accessed 19 Feb 2015.

Yamanoi, T. (2008). Analysis of Japanese Biology 2 textbooks and the introduction of a student experiment with origami birds (Avis papyrus) aiming at further understanding of the mechanism of evolution. Japanese Journal of Biological Education, 48(4), 197-210. (in Japanese with English abstract).

Yamanoi, T. (2010). "Origami bird (Avis papyrus)", a teaching material about evolution by natural selection: ideas of further improvement inferred from student's experiment. Iden, 64, 96-103. (in Japanese).

Yamanoi, T., Sakura, O., Suzuki, K., \& Takemura, M. (2011). Effects of "Biology II" class on high school students' understanding about evolution. Japanese Journal of Biological Education, 52(1,2), 28-37. (in Japanese with English abstract).

Yamanoi, T., Suzuki, K., Takemura, M., \& Sakura, O. (2012a). Improved "origami bird" protocol enhances Japanese students' understanding of evolution by natural selection-a novel approach linking DNA alteration to phenotype change-. Evolution: Education \& Outreach, 5, 292-300.

Yamanoi, T., Takemura, M., Sakura, O., \& Kazama, T. (2012b). Development and evaluation of an activity to teach molecular phylogeny, deep time and classification systems to Japanese high school students. Asian Journal of Biology Education, 6, 13-25.

Received: 2 March 2015 Accepted: 1 August 2015

Published online: 11 August 2015 